\title{
Modeling of Thermal Distributions around a Barrier at the Interface of Coating and Substrate
}

\author{
Ali Sahin \\ Department of Mathematics, Fatih University, Buyukcekmece, 34500 Istanbul, Turkey \\ Correspondence should be addressed to Ali Sahin; asahin@fatih.edu.tr
}

Received 7 March 2013; Accepted 2 August 2013

Academic Editor: Adem Kilıçman

Copyright ( 2013 Ali Sahin. This is an open access article distributed under the Creative Commons Attribution License, which permits unrestricted use, distribution, and reproduction in any medium, provided the original work is properly cited.

\begin{abstract}
Due to constant heat flux, the thermal distribution around an insulated barrier at the interface of substrate and functionally graded material (FGM) which are essentially two-phase particulate composites is examined in such a way that the volume fractions of the constituents vary continuously in the thickness direction. Using integral transform method, two-dimensional steady-state diffusion equation with variable conductivity is turned into constant coefficient differential equation. Reducing that equation to a singular integral equation with Cauchy type, the temperature distribution around the barrier is obtained by defining an unknown function, which is called density function, as a series expansion of orthogonal polynomials. Results are shown for different thickness and nonhomogeneity parameters of FGM.
\end{abstract}

\section{Introduction}

There are many engineering applications under severe thermal loading that require high temperature resistant materials in various forms of composites and bonded materials such as power generation, transportation, aerospace, and thermal barrier coatings. New developments in science and technology rely on the developments of new materials. Composites appear to provide the necessary flexibility in the design of these new materials, which are essentials that every part of the material in use exhibits uniform properties. In the mid-1980s, a new composite material, which was initially designed as a kind of thermal barrier coating used in aerospace structural applications and fusion reactors, was found by a group of scientists in Japan. Because of the material's structure, it is named as functionally graded material (FGM). FGMs were used in modern technologies as advanced structures where the composition or the microstructure is locally varied so that a certain variation of the local material properties is achieved [1]. FGMs are also developed for general use as structural components in extremely high-temperature environments. The concept is to make a composite material by varying the microstructure from one material to another material with a specific gradient. The transition between the two materials can usually be approximated by means of a power series or an exponential function [2-5].

The aircraft and aerospace industry and the computer circuit industry are very interested in the possibility of materials that can withstand very high thermal gradients. This is normally achieved by using a ceramic layer connected with a metallic layer. The composition profile which varies from $0 \%$ ceramic at the interface to $100 \%$ ceramic near the surface, in turn, is selected in such a way that the resulting nonhomogeneous material exhibits the desired thermomechanical properties. The concept of FGMs could provide great flexibility in material design by controlling both composition profile and microstructure [6].

A number of reviews dealing with various aspects of FGMs have been published in the past few decades. They show that most of early research studies in FGMs had focused more on thermal stress analysis and fracture mechanics. Fracture mechanics of FGMs have been studied analytically by Erdogan and coworkers [7-9]. Erdogan identified a number of typical problem areas relating to the fracture of FGMs by considering mainly the investigation of the nature of stress singularity near the tip of a crack in a different geometry [10]. Erdogan also investigated the nature of the crack-tip stress field in a nonhomogeneous medium having a shear 
modulus with a discontinuous derivative. The problem was considered for the simplest possible loading and geometry, namely, the antiplane shear loading of two bonded half spaces, in which the crack is perpendicular to the interface. It was shown that the square-root singularity of the crack-tip stress field is unaffected by the discontinuity in the derivative of the shear modulus [11]. Related to the fracture problems in composite materials, the solution of integral equations with strongly singular kernels is examined by Kaya and Erdogan $[12,13]$. In an axisymmetric coordinate system, an embedded axisymmetric crack in a nonhomogeneous infinite medium was studied by Ozturk and Erdogan [14]. They showed the effect of the material nonhomogeneity on the stress intensity factors under constant Poisson's ratio.

Due to the material mismatch at the interface of substrate and coating, the thermal distributions and thermal stresses on the crack or insulated barrier at the interface are examined by researchers. A general analysis of one-dimensional steadystate thermal stresses in a hollow thick cylinder made of functionally graded material is developed by Jabbari et al. [15]. The material properties were assumed to be nonlinear with a power law distribution. The mechanical and thermal stresses were obtained through the direct method of solution of the Navier equation. The problem of general solution for the mechanical and thermal stresses in a short length functionally graded hollow cylinder due to the two-dimensional axisymmetric steady-state loads was solved using the Bessel functions by Jabbari et al. [16]. A standard method was used to solve a nonhomogeneous system of partial differential Navier equations with nonconstants coefficients, using Fourier series.

Jin and Noda [17] examined an internal crack problem in nonhomogeneous half-plane under thermal loading using the airy stress function method and Fourier transform. They reduced the problem to a system of singular integral equations and solved it by numerical methods. They used superposition method by defining the problem in two cases. One is the linear one-dimensional heat conduction problem under constant heat flux without crack, and the other one is the two-dimensional heat conduction problem with an insulated crack subject to constant heat flux which is acted in the opposite direction. It was also considered the problem of an axisymmetric penny-shaped crack embedded in an isotropic graded coating bonded to a semi-infinite homogeneous medium by Rekik et al. [18]. The coating's material gradient is parallel to the axisymmetric direction and is orthogonal to the crack plane. They used Hankel transform to convert the equations into coupled singular integral equations along with the density function, and they solved it numerically.

In this study, the Hankel integral transform method will be used to solve the heat equation in axisymmetric coordinate system. Problem will be examined as a one-dimensional and a two-dimensional heat conduction problem that is a mixed boundary value problem over the real line. Using mixed boundary conditions a Fredholm integral equation will be obtained with Cauchy type singularity and then it will be solved by using some known numerical techniques $[19,20]$.

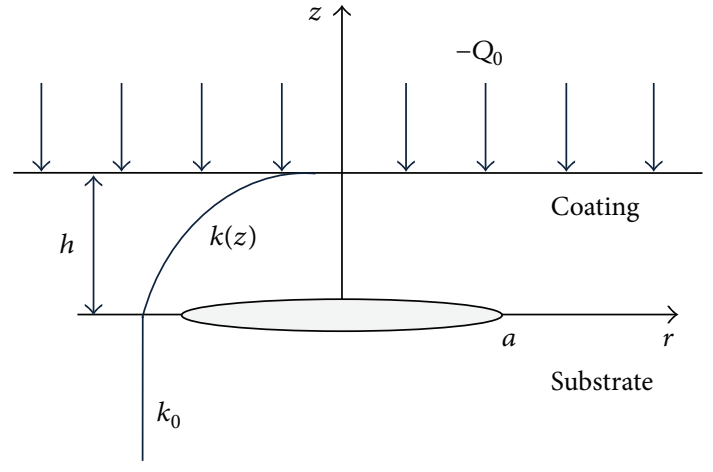

FIgURE 1: Geometry of the heat conduction problem.

\section{Definition of the Problem}

The thermal distribution around a penny-shaped barrier at the interface of graded composite coating and a substrate is given by the following steady-state heat equation in axisymmetric coordinate system:

$$
\frac{1}{r} \frac{\partial}{\partial r}\left(r k(z) \frac{\partial T}{\partial r}\right)+\frac{\partial}{\partial z}\left(k(z) \frac{\partial T}{\partial z}\right)=0
$$

and the conductivities of the substrate and the graded composite coating are, respectively, given as

$$
\begin{gathered}
k(z)=k_{0}, \quad z \leq 0, \\
k(z)=k_{0} e^{\delta z}, \quad z \geq 0,
\end{gathered}
$$

where $k_{0}$ is a constant and $\delta$ is the nonhomogeneity parameter related to the graded coating. Note that the conductivity is continuous at the interface of substrate and the graded composite coating. As shown in Figure 1, it is considered a penny-shaped barrier with radius $a$, centered at the origin of the axisymmetric coordinate system. It is assumed that a uniform heat flux is applied over the stress free boundary, and the barrier faces remain insulated.

The solution can be obtained using superposition method which is an addition of one- and two-dimensional heat conduction problems, $T_{1}(z)$ and $T_{2}(r, z)$, respectively, as shown in Figures 2(a) and 2(b). As in Figure 2(a), it will be assumed that there will be no barrier and in flux causes thermal distribution only $z$ direction. On the other hand, in Figure 2(b), flux will be assumed in an opposite direction on the barrier that causes thermal distribution in $(r, z)$ plane.

Rewriting (1) assuming that no changing in $r$ direction:

$$
\begin{gathered}
\delta \frac{d T_{1}}{d z}+\frac{d^{2} T_{1}}{d z^{2}}=0, \quad 0 \leq z \leq h ; \\
\frac{d^{2} T_{1}}{d z^{2}}=0, \quad z \leq 0
\end{gathered}
$$




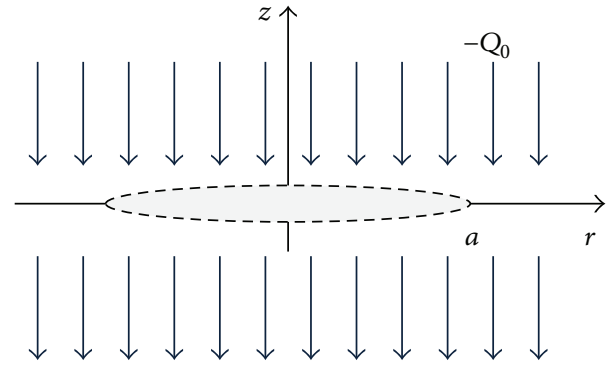

(a)

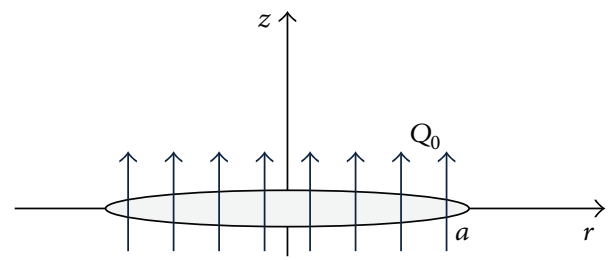

(b)

Figure 2: (a) One-dimensional heat conduction without an insulated barrier, (b) Two-dimensional heat conduction with an insulated barrier.

along with suitable boundary conditions:

$$
\begin{aligned}
k(z) \frac{d}{d z} T_{1}(z) \longrightarrow \text { finite, } \quad z \longrightarrow-\infty \\
k(z) \frac{d}{d z} T_{1}(z)=-Q_{0}, \quad z=h, \\
T_{1}\left(0^{+}\right)=T_{1}\left(0^{-}\right),
\end{aligned}
$$

the solution of the one-dimensional heat conduction is obtained straightforward as

$$
T_{1}(z)= \begin{cases}\frac{Q_{0}}{k_{0} \delta} e^{-\delta z}, & 0 \leq z \leq h, \\ \frac{Q_{0}}{k_{0} \delta}, & z \leq 0,\end{cases}
$$

where the continuity can be seen as $z \rightarrow 0$. For twodimensional heat conduction problem, (1) can be simplified for $0<z \leq h$ as

$$
\begin{gathered}
\frac{\partial^{2}}{\partial r^{2}} T_{2}(r, z)+\frac{1}{r} \frac{\partial}{\partial r} T_{2}(r, z)+\frac{\partial^{2}}{\partial z^{2}} T_{2}(r, z) \\
+\delta \frac{\partial}{\partial z} T_{2}(r, z)=0,
\end{gathered}
$$

and for $z<0$,

$$
\frac{\partial^{2}}{\partial r^{2}} T_{2}(r, z)+\frac{1}{r} \frac{\partial}{\partial r} T_{2}(r, z)+\frac{\partial^{2}}{\partial z^{2}} T_{2}(r, z)=0
$$

with standard boundary conditions:

$$
\begin{array}{cl}
k(z) \frac{\partial}{\partial z} T_{2}(r, z) \longrightarrow \text { finite, }, & z \longrightarrow-\infty, \\
k(z) \frac{\partial}{\partial z} T_{2}(r, z)=0, \quad z=h, & 0 \leq r<\infty, \\
\frac{\partial}{\partial z} T_{2}\left(r, 0^{+}\right)=\frac{\partial}{\partial z} T_{2}\left(r, 0^{-}\right), & a<r<\infty,
\end{array}
$$

and mixed boundary conditions:

$$
\begin{gathered}
k(z) \frac{\partial}{\partial z} T_{2}(r, z)=Q_{0}, \quad z \longrightarrow 0,0 \leq r<a, \\
T_{2}\left(r, 0^{+}\right)=T_{2}\left(r, 0^{-}\right), \quad a<r<\infty .
\end{gathered}
$$

Equation (6) will be solved using Hankel integral transform such that $T_{2}(r, z)$ denote the Hankel transform of zero order and $\tau(\rho, z)$ denote inverse Hankel transform of zero order [21] shown as below, respectively,

$$
\begin{aligned}
& T_{2}(r, z)=\int_{0}^{\infty} \tau(\rho, z) J_{0}(r \rho) \rho d \rho \\
& \tau(\rho, z)=\int_{0}^{\infty} T_{2}(r, z) J_{0}(r \rho) r d r .
\end{aligned}
$$

Using Hankel transform, the solution of (6) along with boundary conditions (8) can be obtained as

$$
\tau(\rho, z)= \begin{cases}A(\rho) e^{-\left(m_{2}+m_{1}\right) z}+A(\rho) \frac{m_{2}+m_{1}}{m_{2}-m_{1}} \\ \times e^{-2 m_{2} h+\left(m_{2}-m_{1}\right) z}, \quad 0<z \leq h, \\ A(\rho) \frac{1}{\rho}\left(e^{-2 m_{2} h}-1\right) & \left(m_{2}-m_{1}\right) \\ \times e^{\rho z}, & -\infty<z<0,\end{cases}
$$

where $m_{1}=\delta / 2, m_{2}=\rho \sqrt{(\delta / 2 \rho)^{2}+1}$ and observing that $m_{2}+m_{1}>0$ and $m_{2}-m_{1}>0$.

\section{Evaluation of Integral Equation}

The unknown value $A(\rho)$ can be obtained by defining a new function, which is called density function [11], such as

$$
\psi(r)=\frac{\partial}{\partial r}\left(T_{2}\left(r, 0^{+}\right)-T_{2}\left(r, 0^{-}\right)\right),
$$

where $\psi(r)$ satisfies the following conditions:

$$
\begin{gathered}
\int_{0}^{a} \psi(r) d r=0, \quad \psi(r)=0, \quad \text { at } a<r<\infty, \\
\psi(r)=-\psi(-r) .
\end{gathered}
$$

Substituting (12) into (13) along with the conditions given in (14), the unknown function $A(\rho)$ can be obtained:

$$
A(\rho)=\frac{1}{F(\rho)} \int_{0}^{a} \psi(s) J_{1}(s \rho) s d s,
$$


where

$$
\begin{aligned}
F(\rho)= & -\rho-\rho\left(\frac{m_{2}+m_{1}}{m_{2}-m_{1}}\right) e^{-2 m_{2} h} \\
& +\left(e^{-2 m_{2} h}-1\right)\left(m_{2}-m_{1}\right) .
\end{aligned}
$$

Using the boundary condition (9) in transformed domain as $z \rightarrow 0^{-}$, it can be obtained as

$$
\int_{0}^{\infty}\left(e^{-2 m_{2} h}-1\right)\left(m_{2}-m_{1}\right) A(\rho) J_{0}(r \rho) \rho d \rho=\frac{Q_{0}}{k_{0}},
$$

and substituting the value of $A(\rho)$ into (17), the integral equation to be solved for unknown $\psi(s)$ can be obtained as

$$
\begin{gathered}
\int_{0}^{a}\left(\int_{0}^{\infty}\left(e^{-2 m_{2} h}-1\right) \eta(\rho) J_{0}(r \rho) J_{1}(s \rho) \rho d \rho\right) \psi(s) s d s \\
\quad=\frac{Q_{0}}{k_{0}},
\end{gathered}
$$

where

$$
\eta(\rho)=\frac{m_{2}-m_{1}}{F(\rho)} .
$$

Defining new normalized variables and parameters such as

$$
\begin{gathered}
s^{\prime}=\frac{s}{a}, \quad r^{\prime}=\frac{r}{a}, \quad \rho^{\prime}=a \rho, \quad h^{\prime}=\frac{h}{a}, \\
\delta^{\prime}=a \delta, \quad \psi(s)=\psi\left(a s^{\prime}\right)=\psi\left(s^{\prime}\right),
\end{gathered}
$$

the integral equation in (18) can be expressed in the form of

$$
\begin{aligned}
\int_{0}^{1} \psi(s) & s d s \int_{0}^{\infty} J_{0}(r \rho) J_{1}(s \rho) \rho d \rho \\
& +\int_{0}^{1} \psi(s) s d s \int_{0}^{\infty} 2 e^{-2 m_{2} h} \eta(\rho) J_{0}(r \rho) J_{1}(s \rho) \rho d \rho \\
& \quad-\int_{0}^{1} \psi(s) s d s \int_{0}^{\infty}(2 \eta(\rho)+1) J_{0}(r \rho) J_{1}(s \rho) \rho d \rho \\
= & q_{0},
\end{aligned}
$$

where $q_{0}=2 Q_{0} / k_{0}$ and the prime sign is removed for the sake of simplicity. The first double integral in (21) can be expressed in terms of the first and second kinds of elliptic integrals, $K$ and $E$, respectively. As $k \rightarrow 1$, the second kind of elliptic integral $E(k)$ has a finite value while the first kind of elliptic integral $K(k)$ has a logarithmic singularity such as

$$
K(k)=\log \left(\frac{4}{\sqrt{1-k^{2}}}\right), \quad E(k)=1 .
$$

Defining a new function $M(s, r)$, given in the appendix, and using some algebraic manipulations, a Cauchy type singularity can obtained by using the properties of $\psi(s)$ in (14). Hence, the first integral of (21) becomes

$$
\begin{aligned}
\int_{0}^{1} \psi(s) & s d s \int_{0}^{\infty} J_{0}(r \rho) J_{1}(s \rho) \rho d \rho \\
= & \frac{1}{\pi} \int_{-1}^{1} \frac{\psi(s)}{s-r} d s \\
& +\frac{1}{\pi} \int_{0}^{1}\left(\frac{M(s, r)-1}{s-r}+\frac{M(s, r)-1}{s+r}\right) \psi(s) d s .
\end{aligned}
$$

The second double integral in (21) has an exponential integrand with negative exponent so that as $\rho \rightarrow \infty$, the integrand asymptotically approaches to zero. The asymptotic expansion of the integrand can be expressed as

$$
\begin{aligned}
e^{-2 h \rho} \rho(-1 & +\frac{h \delta^{2}-\delta}{2^{2} \rho}-\frac{h^{2} \delta^{4}-2 h \delta^{3}}{2^{5} \rho^{2}} \\
& +\frac{(1 / 3) h^{3} \delta^{6}-h^{2} \delta^{5}-2 h \delta^{4}+2 \delta^{3}}{2^{7} \rho^{3}} \\
& -\frac{(1 / 3) h^{4} \delta^{8}+(4 / 3) h^{3} \delta^{7}-8 h^{2} \delta^{6}-40 h \delta^{5}}{2^{11} \rho^{4}} \\
& \left.+O\left(\rho^{-5}\right)\right) .
\end{aligned}
$$

Since there is no singularity and any discontinuity over the interval, and due to the asymptotic expansion as $\rho \rightarrow \infty$, the infinite integral can be approximated over the interval $[0, B]$. Depending on parameters $h$ and $\delta$, the value of $B$ can be chosen large enough to obtain small enough value of the integral (less than $10^{-25}$ ) over $[B, \infty$ ). Hence, the second double integral in (21) may be expressed as

$$
\begin{aligned}
\int_{0}^{1} \psi(s) s d s \int_{0}^{\infty} 2 e^{-2 m_{2} h} \eta(\rho) J_{0}(r \rho) J_{1}(s \rho) \rho d \rho \\
\quad=\int_{0}^{1} \psi(s) s d s \int_{0}^{B(h, \delta)} 2 e^{-2 m_{2} h} \eta(\rho) J_{0}(r \rho) J_{1}(s \rho) \rho d \rho .
\end{aligned}
$$

Finally, the last integral in (21) can be evaluated by using a series

$$
\begin{aligned}
G_{A}(\rho)= & -\frac{\delta}{2^{2}}+\frac{\delta^{3}}{2^{6}} \frac{1}{\rho^{2}}-\frac{\delta^{5}}{2^{9}} \frac{1}{\rho^{4}}+\frac{5 \delta^{7}}{2^{14}} \frac{1}{\rho^{6}} \\
& -\cdots+\frac{429 \delta^{15}}{2^{30}} \frac{1}{\rho^{14}}-O\left(\rho^{-16}\right),
\end{aligned}
$$

an asymptotic expansion of the integrand $G(\rho)=\rho(2 \eta(\rho)+$ 1) as $\rho \rightarrow \infty$. Let us define a value $C$ that depends on 
parameters $h$ and $\delta$ and superposes the infinite integral as follows:

$$
\begin{aligned}
\int_{0}^{\infty} G(\rho) J_{0}(r \rho) J_{1}(s \rho) d \rho \\
=\int_{0}^{C(h, \delta)} G(\rho) J_{0}(r \rho) J_{1}(s \rho) d \rho \\
\quad \times \int_{C(h, \delta)}^{\infty} G_{A}(\rho) J_{0}(r \rho) J_{1}(s \rho) d \rho \\
\quad+\int_{C(h, \delta)}^{\infty}\left[G(\rho)-G_{A}(\rho)\right] J_{0}(r \rho) J_{1}(s \rho) d \rho .
\end{aligned}
$$

For a particular value of $C, G(C)-G_{A}(C) \approx 0$ can be obtained; then the last infinite integral can be expressed as

$$
\begin{aligned}
\int_{0}^{\infty} G & (\rho) J_{0}(r \rho) J_{1}(s \rho) d \rho \\
= & \int_{0}^{C(h, \delta)} G(\rho) J_{0}(r \rho) J_{1}(s \rho) d \rho \\
& +\int_{C(h, \delta)}^{\infty} G_{A}(\rho) J_{0}(r \rho) J_{1}(s \rho) d \rho .
\end{aligned}
$$

Since the function $G(\rho)$ is a smooth function over the interval $[0, C]$, it can be evaluated numerically using Gaussian quadrature along with Chebyshev orthogonal polynomials. On the other hand, the evaluation of the integral over the interval $[C, \infty)$ can be evaluated separately for each term in $G_{A}(\rho)$ like

$$
\begin{aligned}
& \int_{C(h, \delta)}^{\infty} G_{A}(\rho) J_{0}(r \rho) J_{1}(s \rho) d \rho \\
& =-\frac{\delta}{2^{2}} \int_{C(h, \delta)}^{\infty} J_{0}(r \rho) J_{1}(s \rho) d \rho \\
& \quad+\int_{C(h, \delta)}^{\infty}\left(\frac{\delta^{3}}{64} \frac{1}{\rho^{2}}-\frac{\delta^{5}}{512} \frac{1}{\rho^{4}}+\frac{5 \delta^{7}}{16384} \frac{1}{\rho^{6}}-\cdots\right) \\
& \quad \times J_{0}(r \rho) J_{1}(s \rho) d \rho,
\end{aligned}
$$

where

$$
\begin{aligned}
H_{0}(r, s) & =\int_{C(h, \delta)}^{\infty} J_{0}(r \rho) J_{1}(s \rho) d \rho \\
& = \begin{cases}\frac{1}{s}-\int_{0}^{C(h, \delta)} J_{0}(r \rho) J_{1}(s \rho) d \rho, & s>r, \\
\frac{1}{2 s}-\int_{0}^{C(h, \delta)} J_{0}(r \rho) J_{1}(s \rho) d \rho, & s=r, \\
-\int_{0}^{C(h, \delta)} J_{0}(r \rho) J_{1}(s \rho) d \rho, & s<r,\end{cases}
\end{aligned}
$$

and the other integrals in (29) can be evaluated iteratively for $k=1,2,3, \ldots$ as follows:

$$
\begin{aligned}
H_{k}(r, s) & =\int_{C(h, \delta)}^{\infty} \frac{J_{0}(r \rho) J_{1}(s \rho)}{\rho^{k}} d \rho \\
& =\frac{J_{0}(r C) J_{1}(s C)}{k C^{k-1}}+\frac{s}{k} L_{k-1}(r, s)+\frac{r}{k} N_{k-1}(r, s),
\end{aligned}
$$

where

$$
\begin{aligned}
L_{k}(r, s) & =\int_{C(h, \delta)}^{\infty} \frac{J_{0}(r \rho) J_{0}(s \rho)}{\rho^{k}} d \rho \\
& =\frac{J_{0}(r C) J_{0}(s C)}{(k-1) C^{k-1}}-\frac{r H_{k-1}(s, r)}{k-1}-\frac{s H_{k-1}(r, s)}{1-k}, \\
N_{k}(r, s) & =\int_{C(h, \delta)}^{\infty} \frac{J_{1}(r \rho) J_{1}(s \rho)}{\rho^{k}} d \rho \\
& =\frac{J_{1}(r C) J_{1}(s C)}{(k+1) C^{k-1}}+\frac{r H_{k-1}(r, s)}{k+1}+\frac{s H_{k-1}(s, r)}{k+1} .
\end{aligned}
$$

The initial values of the integral $H_{1}(r, s), L_{2}(r, s)$, and $N_{3}(r, s)$ are shown in the appendix.

\section{Numerical Evaluation of Integral Equation}

The integral equation in (21) can be solved using Gaussian quadrature method. Using the condition given in (14), the unknown function $\psi(s)$ can be defined in terms of the truncated $N$ th term series expansion of Chebyshev orthogonal polynomials of the first kind, $T_{n}(s)$, as

$$
\psi(s)=\sum_{n=1}^{N} A_{2 n-1} \frac{T_{2 n-1}(s)}{\sqrt{1-s^{2}}}, \quad-1<s<1,
$$

where $A_{2 n-1}$ are coefficients. Substituting the series expansion of the unknown function $\psi(s)$ into the first integral in (23), it can be found that

$$
\begin{aligned}
\frac{1}{\pi} \int_{-1}^{1} \frac{\psi(s)}{s-r} d s & =\sum_{n=1}^{N} A_{2 n-1} \frac{1}{\pi} \int_{-1}^{1} \frac{T_{2 n-1}(s)}{(s-r) \sqrt{1-s^{2}}} d s \\
& =\sum_{n=1}^{N} A_{2 n-1} U_{2 n-2}(r),
\end{aligned}
$$


where $U_{2 n-2}(r)$ is the Chebyshev polynomials of the second kind, and eliminating the logarithmic singularities, shown in appendix, the second integral in (23) can be written as

$$
\begin{aligned}
& \frac{1}{\pi} \int_{0}^{1}(\left.\frac{M(s, r)-1}{s-r}+\frac{M(s, r)-1}{s+r}\right) \psi(s) d s \\
&= \sum_{n=1}^{N} A_{2 n-1} \frac{1}{\pi} \\
& \quad \times \int_{0}^{1}\left(\frac{M(r, s)-1}{s-r}+\frac{M(r, s)-1}{s+r}+\frac{1}{2 r} \log \left|\frac{s-r}{s+r}\right|\right) \\
& \quad \times \frac{T_{2 n-1}(s)}{\sqrt{1-s^{2}}} d s .
\end{aligned}
$$

Finally, substituting the truncated series representation of $\psi(s)$ into (25) and (29) system of algebraic equation can be obtained the to be solved for $A_{2 n-1}$,

$$
\begin{gathered}
\sum_{n=1}^{N} A_{2 n-1}\left(U_{2 n-2}\left(r_{i}\right)+\frac{1}{2 r_{i}} \frac{T_{2 n-1}\left(r_{i}\right)}{2 n-1}+Z_{1}\left(r_{i}\right)\right. \\
\left.+Z_{2}\left(r_{i}\right)-Z_{3}\left(r_{i}\right)\right)=q_{0},
\end{gathered}
$$

where $r_{i}, i=1,2,3, \ldots$, are collocation points and

$$
\begin{aligned}
& Z_{1}\left(r_{i}\right)= \frac{1}{\pi} \int_{0}^{1}\left(\frac{M\left(r_{i}, s\right)-1}{s-r_{i}}+\frac{M\left(r_{i}, s\right)-1}{s+r_{i}}\right. \\
&\left.+\frac{1}{2 r_{i}} \log \left|\frac{s-r_{i}}{s+r_{i}}\right|\right) \frac{T_{2 n-1}(s)}{\sqrt{1-s^{2}}} d s \\
& Z_{2}\left(r_{i}\right)=\int_{0}^{1}\left(\int_{0}^{B(h, \delta)} 2 e^{-2 m_{2} h} \eta(\rho) J_{0}\left(r_{i} \rho\right) J_{1}(s \rho) \rho d \rho\right) \\
& \times \frac{s T_{2 n-1}(s)}{\sqrt{1-s^{2}}} d s, \\
& Z_{3}\left(r_{i}\right)= \int_{0}^{1}\left(\int_{0}^{C(h, \delta)} G(\rho) J_{0}\left(r_{i} \rho\right) J_{1}(s \rho) d \rho\right. \\
& \times \frac{s T_{2 n-1}(s)}{\sqrt{1-s^{2}}} d s .
\end{aligned}
$$

\section{Results}

Because of the nature of the problem it is necessary to increase the density of the collocation points near the ends $r= \pm 1$. Thus, these points may be selected as follows:

$$
T_{n}\left(r_{i}\right)=0, \quad r_{i}=\cos \left(\frac{(2 i-1) \pi}{2 N}\right), \quad i=1,2, \ldots, N .
$$

Then, we get a $(N \times N)$ system of equations whose solution gives the coefficients $A_{2 n+1}$. With known coefficient values, the temperature distribution around the insulated barrier may be obtained by integrating (13) such as

$$
\begin{aligned}
T_{2}\left(r, 0^{+}\right)-T_{2}\left(r, 0^{-}\right) \\
\quad=\sum_{n=1}^{N} A_{2 n-1} \int_{-1}^{r / a} \frac{a T_{2 n-1}(s)}{\sqrt{1-s^{2}}} d s .
\end{aligned}
$$

Defining new variable like

$$
s=\cos \theta, \quad \pi \leq \theta \leq \arccos \left(\frac{r}{a}\right),
$$

the integral in (40) can be evaluated using the relation

$$
U_{n}(t)=\frac{\sin \{(n+1) \arccos t\}}{\sin (\arccos t)},
$$

and the difference in temperature distribution on the plane of the insulated barrier can be obtained as

$$
\begin{aligned}
T^{*}(r) & =\frac{T_{2}\left(r, 0^{+}\right)-T_{2}\left(r, 0^{-}\right)}{a} \\
& =-\sqrt{1-\left(\frac{r}{a}\right)^{2}} \sum_{n=1}^{N} A_{2 n-1} \frac{U_{2 n-2}(r / a)}{2 n-1} .
\end{aligned}
$$

\section{Appendix}

The function $M(s, r)$ in (23) can be defined [14] as

$$
M(s, r)= \begin{cases}\frac{r}{s} E\left(\frac{s}{r}\right)+\frac{s^{2}-r^{2}}{r s} K\left(\frac{s}{r}\right), & s<r, \\ E\left(\frac{r}{s}\right), & s>r,\end{cases}
$$

in terms of the complete elliptic integrals of the first and second kinds, respectively,

$$
\begin{gathered}
K(k)=\int_{0}^{\pi / 2} \frac{d \theta}{\sqrt{1-k^{2} \sin ^{2} \theta}}, \\
E(k)=\int_{0}^{\pi / 2} \sqrt{1-k^{2} \sin ^{2} \theta} d \theta .
\end{gathered}
$$


For $k \geq 2$, the values of the integrals in (31), (32), and (33), respectively, can be obtained by solving the initial value of each integral for $k=1$ such that

$$
\begin{aligned}
H_{1}(r, s) & =\int_{C(h, \delta)}^{\infty} \frac{J_{0}(r \rho) J_{1}(s \rho)}{\rho} d \rho \\
& =\frac{1}{\pi} \int_{0}^{\pi} \frac{s-r \cos \phi}{R}\left(1+J_{1}(R C(h, \delta))\right. \\
L_{1}(r, s) & =\int_{C(h, \delta)}^{\infty} \frac{J_{0}(r \rho) J_{0}(s \rho)}{\rho} d \rho \\
& =\frac{1}{\pi} \int_{0}^{\pi}\left(-\gamma-\log \left(\frac{R C(h, \delta)}{2}\right)\right. \\
N_{1}(r, s) & \left.=\int_{C(h, \delta)}^{\infty} \frac{J_{1}(r \rho) J_{1}(s \rho)}{\rho} d \rho\right) d \phi, \\
& =\frac{r s}{\pi} \int_{0}^{\pi} \frac{\sin ^{2} \phi}{R}\left(\int_{C(h, \delta)}^{\infty} J_{1}(R \rho) d \rho\right) d \phi,
\end{aligned}
$$

where $R^{2}=r^{2}+s^{2}-2 r s \cos \phi$.

Due to the logarithmic singularity as $s \rightarrow r$ in (23), the ratio $((M(s, r)-1) /(s-r)) \rightarrow(0 / 0)$ has undetermined limiting case. Using (22) along with L'Hospital's rule, we have

$$
\lim _{s \rightarrow r} \frac{M(s, r)-1}{s-r}=-\frac{1}{2 r} \log |s-r|+\frac{1}{r}(\log \sqrt{8 r}-1) .
$$

Now, by adding and subtracting the leading part of the logarithmic function,

$$
\begin{gathered}
\frac{1}{\pi} \int_{0}^{1}\left(\frac{M(r, s)-1}{s-r}+\frac{M(r, s)-1}{s+r}+\frac{1}{2 r} \log |s-r|\right) \psi(s) d s \\
-\frac{1}{2 \pi r} \int_{0}^{1}(\log |s-r|) \psi(s) d s,
\end{gathered}
$$

and using the symmetry property of $\psi(s)$ like

$$
\begin{aligned}
-\int_{0}^{1}(\log |s-r|) \psi(s) d s= & -\int_{-1}^{1}(\log |s-r|) \psi(s) d s \\
& -\int_{0}^{1}(\log |s+r|) \psi(s) d s
\end{aligned}
$$

we have

$$
\begin{gathered}
\frac{1}{\pi} \int_{0}^{1}\left(\frac{M(r, s)-1}{s-r}+\frac{M(r, s)-1}{s+r}+\frac{1}{2 r} \log \left|\frac{s-r}{s+r}\right|\right) \psi(s) d s \\
-\frac{1}{\pi} \int_{-1}^{1} \frac{1}{2 r}(\log |s-r|) \psi(s) d s,
\end{gathered}
$$

where

$$
\frac{1}{\pi} \int_{-1}^{1} \log |s-r| \frac{T_{2 n-1}(s)}{\sqrt{1-s^{2}}} d s=-\frac{T_{2 n-1}(r)}{2 n-1},
$$

using the series expansion of unknown function $\psi(s)$ in (34).

\section{Acknowledgment}

This work is supported by the Scientific Research Fund of Fatih University under Project number P50040801-2.

\section{References}

[1] H.-S. Shen, Functionally Graded Materials Nonlinear Analysis of Plates and Shells, CRC Press, New York, NY, USA, 2009.

[2] S. Suresh and A. Mortensen, Fundamentals of Functionally Graded Materials, Institute of Materials Communications Limited, London, UK, 1998.

[3] K. Trumble, Functionally Graded Materials 2000, Ceramic Transactions 114, The American Ceramics Society, Westerville, Ohio, USA, 2001.

[4] W. Pan, Functionally Graded Materials VII, Trans Tech Publications LTD, Zürich, Switzerland, 2003.

[5] O. van der Biest, FGM, 2004, Functionally Graded Materials VIII, Trans Tech. Publications LTD, Zürich, Switzerland, 2005.

[6] I. Shiota and Y. Miyamoto, Functionally Graded Materials 1996, Elsevier, New York, NY, USA, 1997.

[7] Y. F. Chen and F. Erdogan, "The interface crack problem for a nonhomogeneous coating bonded to a homogeneous substrate," Journal of the Mechanics and Physics of Solids, vol. 44, no. 5, pp. 771-787, 1996.

[8] A. Şahin, "An interface crack between a graded coating and a homogeneous substrate," Turkish Journal of Engineering and Environmental Sciences, vol. 28, no. 2, pp. 135-148, 2004.

[9] A. Sahin and F. Erdogan, "On debonding of graded thermal barrier coatings," International Journal of Fracture, vol. 129, no. 4, pp. 341-359, 2004.

[10] F. Erdogan, "Fracture mechanics of functionally graded materials," Composites Engineering, vol. 5, no. 7, pp. 753-770, 1995.

[11] F. Erdogan, "The crack problem for bonded nonhomogeneous materials under antiplane shear loading," ASME Journal of Applied Mechanics, vol. 52, pp. 823-828, 1995.

[12] A. Kaya and F. Erdogan, "On the solution of integral equations with a strongly singular kernels," Quarterly of Applied Mathematics, vol. 45, pp. 105-122, 1987.

[13] A. Kaya and F. Erdogan, "On the solution of integral equations with a generalized cauchy kernel," Quarterly of Applied Mathematics, vol. 45, pp. 455-469, 1987.

[14] M. Ozturk and F. Erdogan, "Axisymmetric crack problem in a nonhomogeneous medium," Journal of Applied Mechanics, Transactions ASME, vol. 60, no. 2, pp. 406-413, 1993. 
[15] M. Jabbari, S. Sohrabpour, and M. R. Eslami, "Mechanical and thermal stresses in a functionally graded hollow cylinder due to radially symmetric loads," International Journal of Pressure Vessels and Piping, vol. 79, no. 7, pp. 493-497, 2002.

[16] M. Jabbari, A. Bahtui, and M. R. Eslami, "Axisymmetric mechanical and thermal stresses in thick short length FGM cylinders," International Journal of Pressure Vessels and Piping, vol. 86, no. 5, pp. 296-306, 2009.

[17] Z. H. Jin and N. Noda, "An internal crack parallel to the boundary of a nonhomogeneous half plane under thermal loading," International Journal of Engineering Science, vol. 31, no. 5, pp. 793-806, 1993.

[18] M. Rekik, M. Neifar, and S. El-Borgi, "An axisymmetric problem of an embedded crack in a graded layer bonded to a homogeneous half-space," International Journal of Solids and Structures, vol. 47, no. 16, pp. 2043-2055, 2010.

[19] F. Erdogan and G. D. Gupta, "Numerical solution of singular integral equations," in Methods of Analysis and Solution of Crack Problems, G. C. Sih, Ed., Martinus Nijhoff Publishers, Dordrecht, The Netherlands, 1973.

[20] F. Erdogan, "Mixed Boundary Value Problems in Mechanics," in Mechanics Today, N. Nasser, Ed., chapter 1, Pergamon Press, Oxford, UK, 1975.

[21] G. N. Watson, A Treatise on the Theory of Bessel Functions, Cambridge University Press, Cambridge, UK, 1966. 


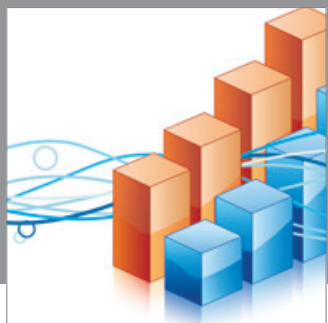

Advances in

Operations Research

mansans

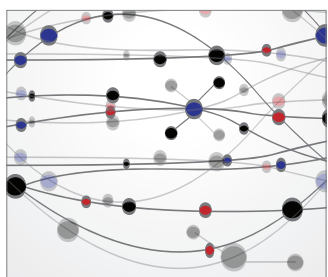

The Scientific World Journal
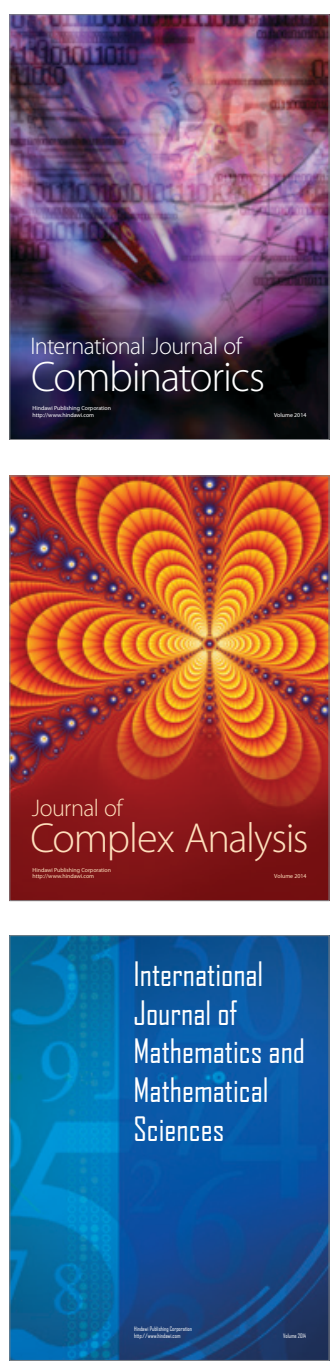
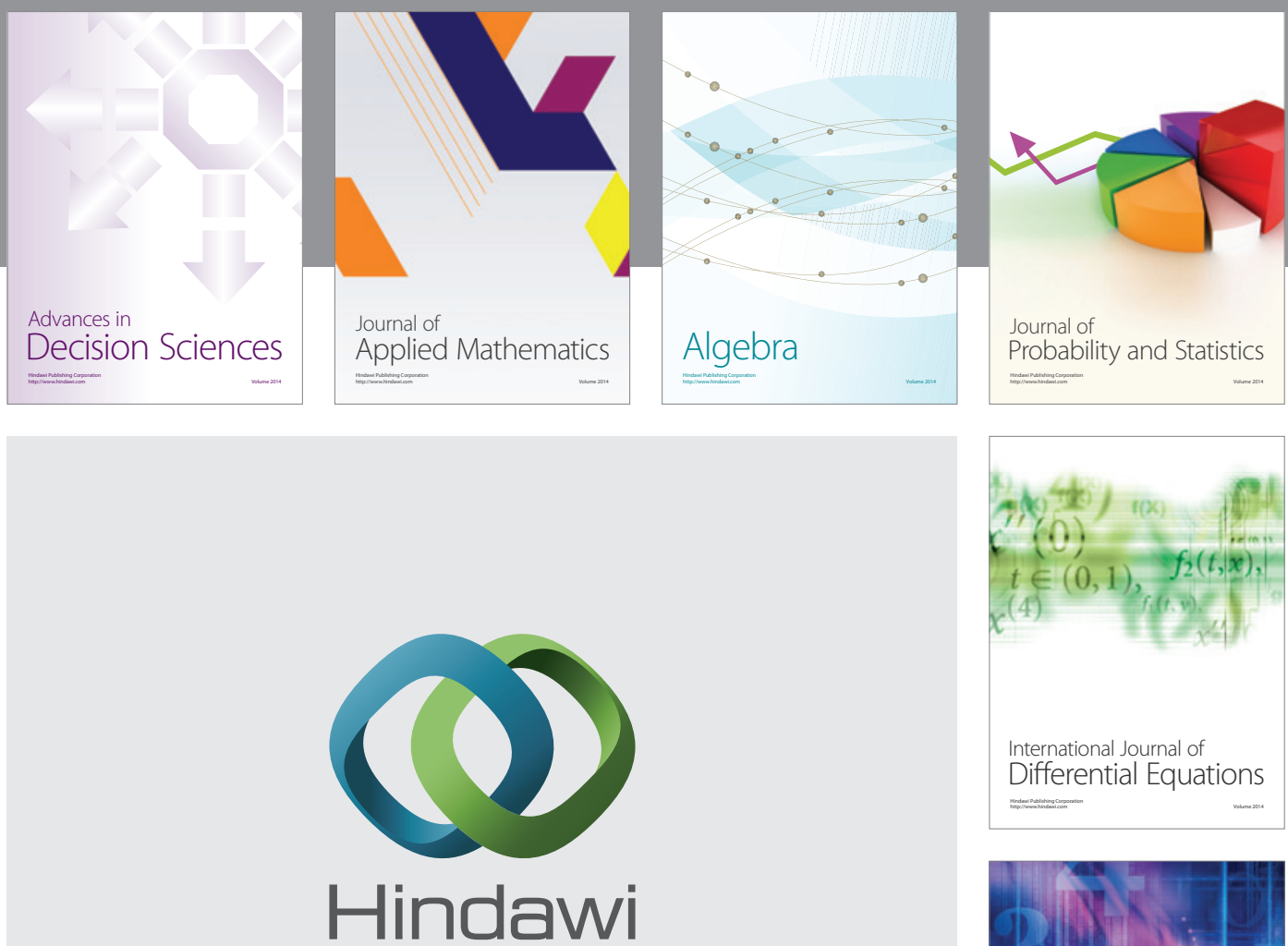

Submit your manuscripts at http://www.hindawi.com
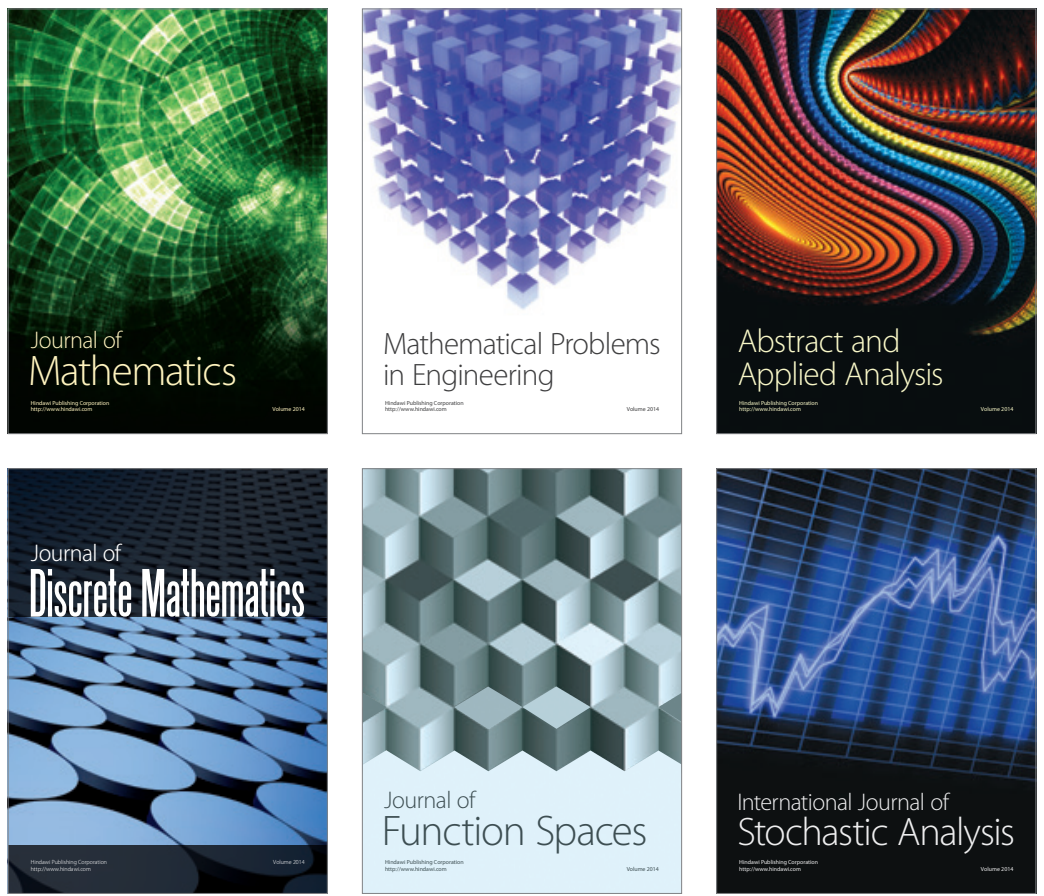

Journal of

Function Spaces

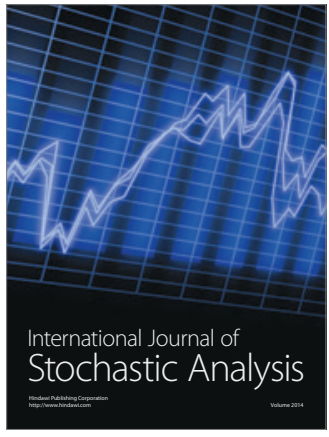

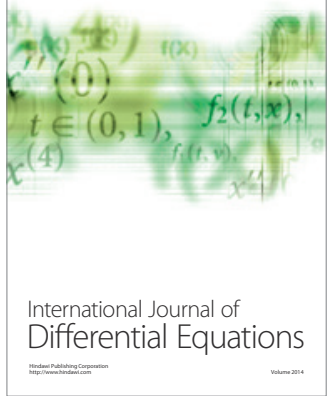
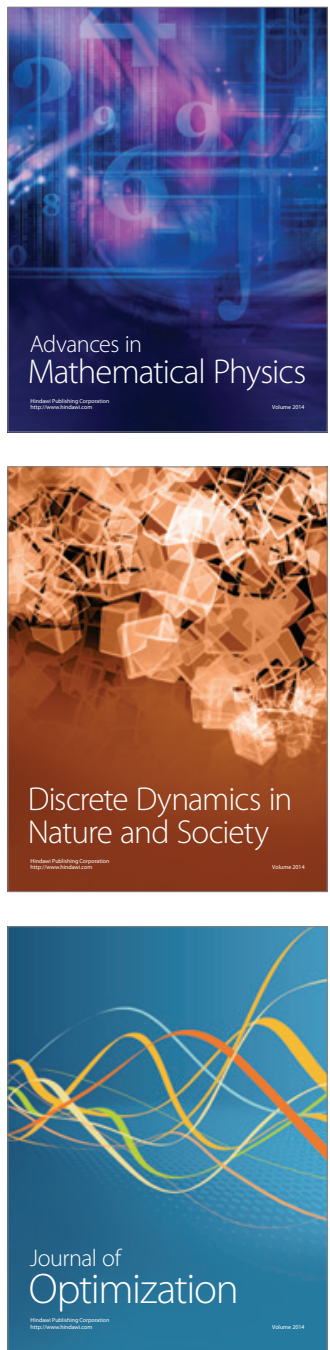